Escola Superior de Tecnologia da Saúde de Coimbra, Rua 5 de Outubro,

3046-854 Coimbra, Portugal GreenUPorto / CiTechCare / LAQV@REQUIMTE - Portugal Asociación de Nutricionistas y Dietistas del Uruguay, Duvimioso Terra 1880 entre Goes y Miguelete 24025101 , Uruguai Facultad Ciencias de la Salud - Universidad Católica de Oriente - Colombia, Sector 3 , Antioguia, Colombia 5 Associação Brasileira de Nustição - Asbran, Rua Claudio Sores, n. 072 onjunto 1607 - Bairro Pinheiros, São Paulo-SP, CEP 05422-0277 Departamento de Salud Nutricional - Ministerio de Salud de Panamá, Antiguo Hospital Gorgas, detrás del Instituto Oncológico, Ancón. Panamá Universidad Juan Agustín Maza Av. Acceso Este, Lat. Sur 2245, Guaymallén, Argentina Friedman School of Nutrition Science and Policy, Tufts

.

Facultad de Ciencias Química Farmacia, Universidad de Guatemala 01012 , Guatem 10 Fresko Perú, Kunan, Lima, Perú Escuela de Nutrición y Antioquia, Calle $70 \mathrm{No} 52$ Apartado Aéreo 1226 Antioquia Colombia

${ }^{2}$ Facultad de Ciencias de la Salud, Universidad Católica de Oriente, Rionegro, Antioquia, Sector 3 , cra. 46 No. 40B 50 Rionegro, Antioquia, Colômbia ${ }^{13}$ Facultad de Ciencias Químicas - Universidad Nacional de Asuncion, Campus .

Universidad Católica de Santa Fe, Cngo. Echagüe 7 S3000 Santa Fe, Argentina Salud, Universidad Privada Norbert Wiener, Av. Arequipa 440. Lima, Perú ${ }^{16}$ Facultad de Ciencias para el Cuidado de la Salud, Universidad San Sebastián, Lientur 1457, Concepción, Bío Bio, Chile Colegio de Profesionales en Nutrición, A008, San José, Granadilla, 11802, Costa Rica 18 Facultad de Ciencias de la Salud, Universidad San Sebastian, Lientur 1457 ,

19 rapcion, Blo Blo, Chile Facultad de Ciencias de la Salud, Universidad Autonom

Faculdade de Ciências da Nutrição e Alimentação da Roberto Frias, 4200-465 Porto Portugal

*Endereço para correspondência:

Ada Rocha

Faculdade de Ciências da Nutrição e Alimentação da Universidade do Porto, Rua Dr. Roberto Frias, 4200-465 Porto, Portuga adarocha@fcna.up.pt

Histórico do artigo:

\section{COMIDA DE RUA: CONSUMO E PERCEÇÃO DE SALUBRIDADE EM CONSUMIDORES PORTUGUESES}

\author{
STREET FOOD: CONSUMPTION AND PERCEPTION OF \\ HYGIENIC STATUS IN PORTUGUESE CONSUMERS
}

João PM Limai, ${ }^{1,2}$; Alfonsina Ortiz ${ }^{3}$; Ángela Velásquez ${ }^{4}$; Beatriz Agazzi ${ }^{3}$; Débora Cabanes ${ }^{5}$; Elka Gonzalez ${ }^{6}$; Emilia Raimondo ${ }^{7}$ Gabriela Fretes ${ }^{8}$ Karla Cordón ${ }^{9}$; Johanna Léon ${ }^{10}$; John Velásquez ${ }^{11}$; Juan Gonzalez ${ }^{12}$; Laura Gonzalez ${ }^{13}$; Luz Arboleda ${ }^{11}$; Maria Celeste Nessier ${ }^{14}$; Marli Brasioli ${ }^{5}$; Saby Mauricio ${ }^{15}$; Samuel Durán ${ }^{16}$; Sonia Ivankovich ${ }^{17}$; Jairo Torres ${ }^{18,19}$; Ada Rocha ${ }^{2,20 *}$

\section{RESUMO}

INTRODUÇÃO: A comida de rua define-se como os alimentos e bebidas prontos a comer, preparados e/ou vendidos na rua ou similares e esta tem-se revelado nos últimos anos uma tendência global na restauração, apesar da insegurança quanto às práticas higio sanitárias percecionada por alguns indivíduos.

OBJETIVOS: Conhecer o perfil de consumidores de comida de rua em Portugal, o tipo de alimentos, a sua frequência de consumo e avaliar a perceção do consumidor em relação à segurança alimentar, por comparação com os resultados obtidos em países da América Latina.

METODOLOGIA: Foi realizado um inquérito online, integrado num estudo multicêntrico a nível ibero-americano.

RESULTADOS: Em Portugal, dos 803 inquiridos, 50,9\% são indivíduos entre os 18-30 anos, e a maioria são do sexo feminino. A maioria dos inquiridos consumiu comida de rua nos três meses anteriores ao preenchimento do inquérito. Cerca de $46 \%$ destes dizem ingeri-la com uma periodicidade inferior a mensal. Os alimentos mais consumidos foram os gelados (89,6\%), as bifanas ou pão com chouriço/ queijo/presunto (83,1\%), as farturas/churros (80,3\%) e os cachorros/hambúrqueres $(79,8 \%)$. Os principais motivos de consumo foram a disponibilidade $(48,7 \%)$ e o sabor (30,3\%). O reporte de episódios de diarreia ou vómito após o consumo de comida de rua em Portugal apresentou um valor muito abaixo da média dos países latino-americanos. Em relação aos locais de venda de comida de rua verifica-se que $80,2 \%$ dos inquiridos considera que os mesmos se encontram sujos ou parcialmente sujos.

CONCLUSÕES: Em Portugal, observa-se um consumo de comida de rua pouco frequente, sendo os gelados e as bifanas os alimentos mais consumidos, e o consumo determinado predominantemente pela disponibilidade e sabor deste tipo de alimentos. Globalmente a perceção de higiene dos consumidores portugueses é má, contudo o reporte de mau estar após a ingestão deste tipo de alimentos foi baixo

\section{PALAVRAS-CHAVE}

Comida de rua, Consumidor, Perceção de segurança

ABSTRACT

INTRODUCTION: Street food is defined as foods and beverages ready-to-eat prepared and / or sold on the street or similar and this has revealed in recent years as global trend in food service, despite the insecurity regarding the hygiene practices perceived by some individuals.

OBJECTIVES: To know the profile of street food consumers in Portugal, the type of food, its frequency of consumption and to evaluate the consumer's perception regarding food safety, comparing to results obtained in Latin American countries. METHODOLOGY: An online survey was conducted as part of a multicenter study at the lberian American level. RESULTS: In Portugal, from 803 inquired individuals, 50.9\% are aged between 18 and 30 years old, and the majority are female. The majority of respondents consumed street food in the three months before answer the questionnaire. About $46 \%$ consumed street food less than monthly. The most consumed foods were ice cream (89.6\%), bifanas or bread with cheese (83.1\%), farturas / churros (80.3\%) and hot-dogs / hamburgers (79.8\%). The main reasons for consumption were availability $(48.7 \%)$ and taste $(30.3 \%)$. The report of vomiting and diarrhea after street food consumption in Portugal is below the average for Latin American countries. About $80.2 \%$ of respondents consider street food vendors as dirty or partially dirty.

CONCLUSIONS: In Portugal, there is a low frequency of consumption of street food, being ice cream and as bifanas the most consumed, and consumption predominantly determined by the availability and taste of this type of food. Overall, the perception of hygiene of Portuguese consumers is bad; however, the report of illness after eating this type of food was low.

KEYWORDS

Street food, Consumers, Safety perception 


\section{INTRODUÇÃO}

De acordo com a definição da Food and Agriculture Organization, a comida de rua define-se como os alimentos e bebidas prontos a comer, preparados e/ou vendidos na rua, em equipamentos móveis ou lojas com apenas um balcão de venda (1). No ranking das cidades do mundo onde a comida de rua é mais popular encontramos exemplos em países da Ásia, África e América, contudo cidades europeias como Bruxelas, Berlim, Londres e Paris assumem-se progressivamente neste âmbito (2). A comida de rua tem-se revelado nos últimos anos uma tendência global na restauração e na forma de fornecer alimentos prontos a comer, vendidos na rua ou outro local público, por vendedores ambulantes. A popularidade do consumo deste tipo de alimentos parece estar relacionada com a urbanização e estilos de vida na qual os consumidores tendem a procurar opções mais baratas (3), a mobilidade dos vendedores, a conveniência da sua localização, preços atrativos e rapidez de preparação (3-5).

Em Portugal, e de acordo com Carlos Costa, na sua visão estratégica do restaurante do futuro, a comida de rua é uma tendência que surge num contexto contemporâneo, informal e simples, e está alinhada com outras, como a dos produtos gourmet e do fast-food saudável. Acrescenta ainda que pode ser apresentada em diversos formatos móveis, como food-trucks, carrinhas, rulotes ou bancas de comida (6). Os espaços de venda ambulante na União Europeia (UE) devem cumprir os requisitos da Comissão Europeia (CE), nomeadamente o Regulamento 852/2004 (7) relativo à higiene dos alimentos; e no que se refere ao Anexo II, cap.III) que estabelece os requisitos para os vendedores de comida de rua. O regulamento horizontal baseia-se no código de prática do Codex Alimentarius relativo aos princípios gerais de higiene (8). Os vendedores de comida de rua devem respeitar as práticas de higiene rigorosas para garantir a segurança do produto acabado. Muitos estudos apresentam discussões sobre as práticas higiénicas de estabelecimentos comerciais de rua localizadas em vários países (3, 4, 8); no entanto, há uma escassez de estudos sobre o consumo e práticas de higiene e a perceção das mesmas em vendedores de comida ambulante na UE.

Adicionalmente, os vendedores de comida de rua devem observar $\mathrm{e}$ cumprir os referenciais sobre venda ambulante em Portugal, incluindo dos Municípios (ex. regime jurídico da venda ambulante-Decreto-Lei 122/79 e atualizações posteriores; Regulamento n. ${ }^{\circ}$ 628/2017 Regulamento Municipal do Comércio a Retalho Não Sedentário de Vila Nova de Gaia).

A segurança da comida de rua é afetada por vários fatores desde a qualidade da matéria prima até às condições de manipulação e armazenamento. A comida de rua pode encontrar-se exposta a condições extremas, tais como a presença de insetos, roedores, animais domésticos e poluição, especialmente em países em vias de desenvolvimento (9). A comida de rua tem sido associada a doenças de origem alimentar maioritariamente devido às dificuldades em assegurar que os alimentos são preparados e vendidos em condições higiénicas, quer em países em vias de desenvolvimento, quer em países desenvolvidos $(10,11)$.

Existe uma perceção generalizada de que a comida de rua é insegura, maioritariamente devido ao ambiente em que esta é preparada, vendida e consumida, sendo exposta contaminação. A comida de rua é encarada como um grande risco de saúde pública devido à falta de infraestruturas e serviços, dificuldades no controle de um grande número de operadores de venda ambulante, devido à sua diversidade, mobilidade e natureza temporária, especialmente em países em vias de desenvolvimento, como a maioria dos pertencentes à América Latina (12).
Atendendo a que a gastronomia se tem vindo a constituir um forte aliado ao nível do Turismo em Portugal (13), e que a comida de rua é parte da oferta gastronómica portuguesa, é importante analisar a perceção dos consumidores de comida de rua uma vez que esta poderá constituir um fator a considerar pelos turistas aquando da decisão de visitar ou re-visitar o país $(14,15)$.

\section{OBJETIVOS}

Conhecer o perfil de consumidores de comida de rua em Portugal, o tipo de alimentos, a sua frequência de consumo e avaliar a perceção do consumidor em relação à segurança alimentar, por comparação com os resultados obtidos em países da América Latina.

\section{METODOLOGIA}

Foi realizado um inquérito online, integrado num estudo multicêntrico a nível ibero americano. O inquérito, composto por 12 perguntas, incluía questões sociodemográficas e de frequência de consumo de 10 alimentos e relato de sintomas de intoxicação alimentar após o consumo destes alimentos e opinião em relação às condições de higiene dos locais de armazenamento (ANEXO 1).

O questionário foi validado pelo método de Delphi, tendo sido ajustado aos alimentos de consumo usual em cada país. O questionário foi difundido online através de diversas plataformas de uso generalizado pela população portuguesa, entre os meses de março e julho do ano de 2016. A seleção dos participantes foi feita por conveniência, uma vez que o questionário foi disseminado online pelos investigadores em plataformas de acesso generalizado e livre, mas condicionadas pelo conhecimento dos mesmos.

\section{RESULTADOS}

\section{Perfil dos inquiridos}

Em Portugal, dos 803 inquiridos, 50,9\% são indivíduos entre os 18-30 anos, e a maioria são do sexo feminino (82,1\%). A maioria dos inquiridos (75,3\%) têm formação superior e 70,2\% habitam numa zona urbana. $61,1 \%$ dos inquiridos trabalham ou estudam em áreas relacionadas com a saúde ou com a ciência dos alimentos.

\section{Caracterização dos consumidores}

A maioria dos inquiridos $(52,2 \%)$ consumiu comida de rua ou de venda ambulante nos três meses anteriores ao preenchimento do inquérito.

\section{Tipo de alimentos e a frequência de consumo em Portugal}

Dos que consumiram comida de rua nos três meses anteriores ao preenchimento do inquérito, apenas $4,0 \%$ a consomem com frequência semanal. Cerca de $46 \%$ dos consumidores de comida de rua portugueses entrevistados dizem ingeri-la com uma periodicidade inferior a mensal (ou seja, menos do que uma vez por mês) (Figura 1). Em Portugal, os alimentos mais consumidos (independentemente da frequência) foram os gelados (89,6\%), as Bifanas ou Pão com chouriço/queijo/presunto $(83,1 \%)$, as farturas/churros $(80,3 \%)$ e os cachorros/hambúrgueres (79,8\%).

Quando analisada a frequência de consumo verificamos que os alimentos mais frequentemente consumidos foram os gelados $(35,9 \%)$, seguidos dos cachorros/hambúrgueres $(31,2 \%)$ e das bifanas/pão com chouriço $(28,1 \%)$.

Entre os alimentos menos consumidos encontramos as sardinhas, a fruta e os pastéis, uma vez que $74,3 \%, 60 \%$ e $51,3 \%$ dos consumidores indicaram não os consumir, respetivamente (Tabela 2). 
Tabela 1

Caracterização sociodemográfica dos consumidores

\begin{tabular}{lcc} 
& N & $\%$ \\
\hline Faixa etária (anos) & & \\
\hline 18 - 30 & 246 & 58,7 \\
\hline 31 - 40 & 82 & 19,6 \\
\hline 41 - 50 & 60 & 14,3 \\
\hline 51 - 60 & 27 & 6,4 \\
\hline$>60$ & 4 & 0,9 \\
\hline Sexo & & \\
\hline Masculino & 78 & 18,6 \\
\hline Feminino & 341 & 81,4 \\
\hline Nível de escolaridade & & \\
\hline Ensino básico & 6 & 1,4 \\
\hline Ensino secundário & 64 & 15,3 \\
\hline Curso de especialização tecnológica & 29 & 6,9 \\
\hline Curso superior (bacharelato/licenciatura) & 190 & 45,3 \\
\hline Pós graduação/mestrado/doutoramento & 130 & 31,0 \\
\hline Zona habitacional & & \\
\hline Rural & 119 & 28,4 \\
\hline Urbana & 300 & 71,6 \\
\hline Área de trabalho/estudo & & \\
\hline Saúde ou Ciências dos Alimentos & 248 \\
\hline Outras & 171 & \\
\hline
\end{tabular}

\section{Figura 1}

Frequência do consumo de comida de rua

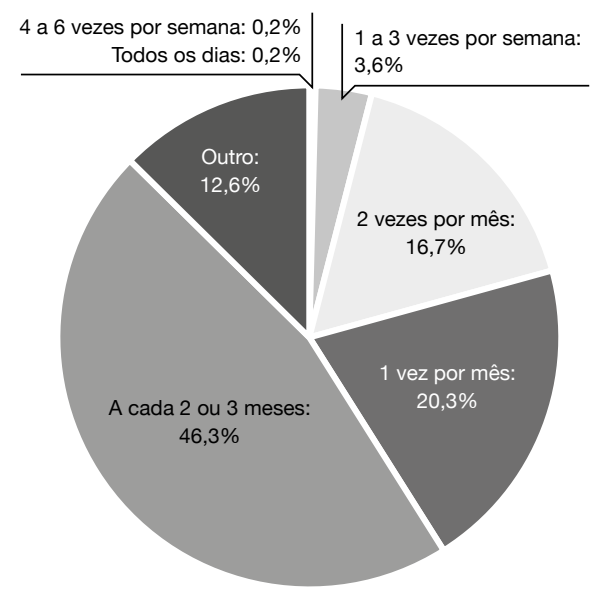

Tabela 2

Frequência de consumo por tipo de alimento

\begin{tabular}{lccc}
\multicolumn{1}{c}{ TIPO DE ALIMENTOS } & \multicolumn{3}{c}{ FREQUENNCIA DE CONSUMO (\%) } \\
& NUNCA & OCASIONALMENTE FREQUENTEMENTE \\
Pastéis & 51,3 & 40,1 & 8,7 \\
\hline Cachorro ou hambúrguer & 20,2 & 48,6 & 31,2 \\
\hline Sardinhas & 74,3 & 21,5 & 4,2 \\
\hline $\begin{array}{l}\text { Bifana/pão com } \\
\text { chouriço/queijo/presunto }\end{array}$ & 16,8 & 55,0 & 28,1 \\
\hline $\begin{array}{l}\text { Tripa ou bolacha } \\
\text { americana }\end{array}$ & 38,3 & 39,3 & 22,4 \\
\hline Farturas ou churros & 19,7 & 59,4 & 20,9 \\
\hline Fruta & 60,0 & 25,4 & 14,5 \\
\hline Doces & 44,0 & 45,4 & 10,5 \\
\hline Crepes ou waffles & 40,4 & 44,7 & 14,9 \\
\hline Gelados & 10,4 & 53,7 & 35,9 \\
\hline
\end{tabular}

Tabela 3

Atitude face a situação de mal-estar após ingestão de comida de rua

\begin{tabular}{lcc}
$\begin{array}{c}\text { ATITUDES EM CASO DE MAL-ESTAR APÓs } \\
\text { INGESTÃO DE COMIDA DE RUA }\end{array}$ & N & $\%$ \\
Procurou o médico & 15 & 46,9 \\
\hline Automedicou-se & 15 & 46,9 \\
\hline Não fez nada & 2 & 6,2 \\
\hline Total & 32 & 100,0 \\
\hline
\end{tabular}

\section{Motivações para o consumo de comida de rua}

Os principais motivos de consumo de comida de rua foram a disponibilidade $(48,7 \%)$ e o sabor $(30,3 \%)$.

\section{Consumo de comida de rua e episódios de diarreia ou vómito}

Em Portugal, 7,6\% dos consumidores referiram alguma vez ter apresentado episódios de diarreia ou vómito depois de consumir alimentos em locais de comida de rua ou venda ambulante. Dos que referem apresentar sintomas de diarreia ou vómito após o consumo de comida de rua, 46,9\% procuraram o médico.

No que se refere à perceção dos inquiridos em relação aos locais de venda de comida de rua ou ambulante verifica-se que $80,2 \%$ considera que os mesmos se encontram sujos ou parcialmente sujos.

\section{DISCUSSÃO DOS RESULTADOS}

O consumo de comida de rua ou de venda ambulante nos três meses anteriores ao preenchimento do inquérito em Portugal apresentou um resultado ligeiramente abaixo da média dos países latino-americanos, onde $54 \%$ dos inquiridos consumiram alimentos de venda ambulante num mesmo período (15).

O consumo de comida de rua em Portugal é muito inferior ao reportado nos países da América Latina, onde $15,8 \%$ dos consumidores indicam ingerir este tipo de alimentos, com a mesma periodicidade (16). Relativamente às motivações para o consumo de alimentos de comida de rua ou venda ambulante, os resultados em Portugal foram concordantes com os encontrados nos países latino-americanos (16). O reporte de episódios de diarreia ou vómito após o consumo de comida de rua ou venda ambulante em Portugal apresentou um valor muito abaixo da média dos países latino americanos, onde 18,2\% dos consumidores referiram já ter apresentado estes sintomas após a ingestão (16). Na América Latina, apenas $26,3 \%$ dos episódios tiveram igual acompanhamento. Nenhum dos países avaliados apresentou uma procura de assistência médica tão significativa como Portugal (16). Apesar do resultado ser alarmante, verifica-se que as condições de higiene consideradas pelos inquiridos em Portugal serão melhores que as apresentadas nos países latino-americanos, uma vez que apenas $8 \%$ dos inquiridos naqueles países consideram que os espaços de venda se encontram limpos (16).

O baixo consumo de comida de rua em Portugal, comparativamente com os restantes países em estudo, poderá estar relacionado com a perceção sobre o estado higio sanitário destes locais, tal como verificado por outros autores (17-19).

Quando questionados acerca do aspeto mais importante a melhorar nos locais de venda ambulante de alimentos, $41,5 \%$ refiram a higiene dos manipuladores de alimentos, 25,5\% a qualidade nutricional dos alimentos e 24,0\% a higiene das instalações, o que revela uma perceção concordante com práticas de higiene observadas em locais de venda de comida de rua já reportados por outros autores em estudos desenvolvidos em Paris (20), Uganda (21), Grécia, Polónia, Tailândia e China (22), Brasil (23). Face ao consumo de comida de rua e a sua importância crescente para o turismo, esforços adicionais 
deverão ser desenvolvidos pelas entidades reguladoras para aumentar o grau de cumprimentos dos requisitos de higiene (24).

O período do ano em que decorreu a recolha de dados poderá ser encarado como uma limitação, uma vez que, devido ao clima e atividades lúdicas decorrentes do mesmo, em Portugal o consumo de comida de rua poderá ser mais influenciado por esse fator do que nos restantes países envolvidos no estudo multicêntrico. Também a metodologia utilizada para a recolha de dados poderá ter conduzido a uma amostragem enviesada com elevada percentagem de indivíduos com elevadas habilitações académicas e constituída por jovens adultos, bem como estudantes ou trabalhadores nas áreas da saúde ou alimentação, por ser também a área de trabalho dos investigadores. A recolha de dados é relativa a episódios passados, pelo que o viés da memória e a influência de notícias ou episódios relatados por outros indivíduos poderão ter influenciados as respostas fornecidas. Em ambas as situações, se ressalva que a opção pela metodologia referida se deveu à uniformização da mesma nos vários países envolvidos no estudo multicêntrico.

\section{CONCLUSÕES}

Em Portugal observa-se um consumo de comida de rua pouco frequente sendo os gelados e as bifanas os alimentos mais consumidos, sendo este determinado predominantemente pela disponibilidade e sabor deste tipo de alimentos.

Globalmente a perceção de higiene dos consumidores portugueses é má, contudo o reporte de mau estar após a ingestão deste tipo de alimentos foi baixo.

As entidades locais em colaboração com a academia deverão trabalharem conjunto para promover a melhoria das condições de venda de comida de rua em Portugal e consequentemente melhorar a perceção do consumidor, bem como ajustando a oferta às expectativas do consumidor.

\section{REFERÊNCIAS BIBLIOGRÁFICAS}

1. World Health Organization. Essential safety requirements for street-vended foods. Food Safety Unit Division of Food and Nutrition. WHO/FNU/FOS/96.7. World Health Organization, Geneva. 1996.

2. Griffin S. World's 23 best cities for street food. 2016.

3. Kraig, B., and C. T. Sen. Street food around the world. An encyclopedia of food and culture. ABC-CLIO, Santa Barbara, CA. 2013.

4. Liu, Z., G. Zhang, and X. Zhang. Urban street foods in Shijiazhuang city, China: current status, safety practices and risk mitigating strategies. Food Control. 2014 41:212-218.

5. Tinker, I. Street foods: traditional micro-enterprise in a modernizing world. Int. J. Polit. Cult. Soc. 2003. 16:331-349.

6. Costa, C. Visão Estratégica e Análise Prospetiva do Restaurante do Futuro. Porto: IDTOUR - Unique Solutions, LDA. e Universidade de Aveiro. 2012.

7. European Parliament and the Council of the European Union. 2004. Commission Regulation (EC) No 852/2004 of the European Parliament and of the Council of 29 April 2004 on the hygiene of foodstuffs. Off. J. Eur. Union L 139:3-21.

8. Hanashiro, A., M. Morita, G. R. Matte, H. M. Matte, and E. A. F. S. Torres. Microbiological quality of selected street foods from a restricted area of São Paulo City, Brazil. Food Control. 2005. 16:439-444.

9. Joint Food and Agriculture Organization of the United Nations- World Health Organization. 2009. Food hygiene. Basic texts, Codex alimentarius, 4th ed. Codex Alimentarius Commission, Rome.

10. Almeida, C. R., Schuch, D. M. T., Gelli, D. S., Cueller, J. A., Diez, A. V., \& Escamilla, J. A. Microbial contamination of street foods sold by street vendors in cities of Latin America. Geneva, Switzerland: World Health Organization. 1996.

11. Bryan, F. L. Risk associated with practices, procedures and processes that lead to outbreaks of foodborne diseases. Journal of Food Protection. 1988. 51, 663-673. 12. Ghosh, M., S. Wahi, M. Kumar, and A. Ganguli. Prevalence of enterotoxigenic Staphylococcus aureus and Shigella spp. in some raw street vended Indian foods. Int. J. Environ. Health Res. 2007. 17:151-156.

13. Bellia, C., Pilato, M., \& Seraphin, H. Street food and food safety: A driver for tourism? Journal of Applied Ceremonial and Communication in Management. 2016. 1, $29-55$. 14. Mak, A. H., Lumbers, M., Eves, A., \& Chang, R. C. An application of the repertory grid method and generalized Procrustes analysis to investigate the motivational factors of tourist food consumption. International Journal of Hospitality Management. 2013. 35, 327-338.

15. Saha, D. Decent work for the street vendors in Mumbai, India e a distant vision. Journal of Workplace Rights. 2009. 14, 220-250.

16. Durán-Agüero S, Arboleda LM, Vargas JEV, Centurión GF, González LE, Céspedes, Rocha A, Lima JPM, Nessier MC, Raimondo EE, Valderrama AMV, Sánchez JCG, Guillén SI, Arrivillaga KRC, Madden EEG, Alza SMM, Cáceres JL, Agazzi B, Ortiz A, Cabanes Débora, Brasioli M, Torres J. Caracterización del consumo de comida callejera según edad, estudio multicéntrico. J. Rev Esp Nutr Hum Diet. 2018; 22(3): 243 - 25. 17. Lertputtarak, $\mathrm{S}$. The relationship between destination image, food image, and revisiting Pattaya, Thailand. International Journal of Business and Management. 2012. 7, 111-121.

18. Yiamjanya, S., \& Wongleedee, K. International tourists' travel motivation by push pull factors and the decision making for selecting Thailand as destination choice. International Journal of Social, Education, Economics and Management Engineering. 2014. 8, 1326-1331.

19. Sirigunna, J. Food safety in Thailand: A comparison between inbound senior and non-senior tourists. Procedia Social and Behavioral Sciences. 2015. 197, p. 2115-2119. 20. Czarniecka-Skubina E, Trafiałek J, Wiatrowski M, and Głuchowski A. An Evaluation of The Hygiene Practices Of European Street Food Vendors and A Preliminary Estimation of Food Safety for Consumers, Conducted In Paris. Journal of Food Protection. 2018. Vol. 81, No. 10, p. 1614-1621.

21. Muyanja, C., L. Nayiga, N. Brenda, and G. Nasinyama. Practices, knowledge and risk factors of street food vendors in Uganda. Food Control. 2011. 22:1551-1558.

22. Trafialek J, Drosinos EH, Laskowski W, Jakubowska-Gawlik K, Tzamalis P, Leksawasdi N, Surawang S, Kolanowski W. Street food vendors' hygienic practices in some Asian and EU countries - A survey. Food Control. 2018. 85: 212 - 222.

23. Cortese, R. D. M., Veirosa, M. B., Feldmanb, C., \& Cavallia, S. B. Food safety and hygiene practices of vendors during the chain of street food production in Florianopolis, Brazil: A cross-sectional study. Food Control. 2016. 62, 178-186.

24. Trafiałek, J., E. H. Drosinos, and W. Kolanowski. Evaluation of street food vendors' hygienic practices using fast observation questionnaire. Food Control. 2017. 80:350-359. 


\section{ANEXO 1}

\section{QUESTIONÁRIO SOBRE CONSUMO DE ALIMENTOS DE VENDA AMBULANTE EM PORTUGAL}

Bom dia/tarde, convidamo-lo a participar no ESTUDO IBERO-AMERICANO SOBRE PERCEÇÃO DO CONSUMO DE COMIDA DE RUA. Solicitamos que responda a este breve questionário de 12 perguntas, que não demora mais de 2 minutos a responder. Informamos que não existem respostas certas ou erradas, boas ou más, e que as suas respostas são confidenciais e só serão utilizadas para este estudo. Solicitamos que seja sincero nas suas respostas, uma vez que isso se refletirá na validade deste estudo. Este questionário só poderá ser respondido por maiores de 18 anos.
1. Idade*
O $18-30$ anos
$31-40$ anos
O 41-50 anos
O $51-60$ anos
O 61 ou mais anos

\section{Sexo* \\ Feminino \\ Masculino}
3. Selecione nível de escolaridade*
O Ensino Básico
O Ensino Secundário
C Curso de Especialização Tecnológica
O Curso Superior (bacharelato, licenciatura)
O Pós-graduação ou superior
4. Mora em:*
O Zona rural
Zona urbana

5. Trabalha ou estuda em áreas relacionadas com a saúde ou ciência dos alimentos?*

O Sim

O Não

6. Consumiu comida de rua ou de venda ambulante nos últimos 3 meses?*

O Sim

O Não

7. Qual a frequência com que consome comida de rua ou de venda ambulante?*

O Todos os dias

O 4-6 vezes por semana

1-3 vezes por semana

O 2 vezes por mês

O 1 vez por mês

O A cada 2 ou 3 meses

O Outro: 
8. Quando come nestes locais, quais são os alimentos que consome com mais frequência?

\begin{tabular}{|l|l|l|l|}
\hline & FREQUENTEMENTE & OCASIONALMENTE & NUNCA \\
\hline Pasteis & & & \\
\hline Cachorro ou Hambúrguer & & & \\
\hline Sardinhas & & & \\
\hline Bifana ou Pão com chouriço/queijo/presunto & & & \\
\hline Tripa ou Bolacha americana & & & \\
\hline Farturas ou Churros & & & \\
\hline Fruta & & & \\
\hline Doces & & & \\
\hline Crepes ou Waffles & & & \\
\hline Gelados & & & \\
\hline
\end{tabular}

9. Qual o principal motivo pelo qual consome estes alimentos? *

Económicos ou baratos

Falta de tempo

Estão disponíveis em locais que frequento

São saborosos

Outro:

10. Alguma vez apresentou episódios de diarreia ou vómitos depois de consumir algum alimento em locais de comida de rua ou de venda ambulante?*

O Sim

O Não

11. Quando isso aconteceu, que atitude tomou?*

Procurou o médico

Automedicou-se

Outro:

12. Segundo a sua perceção a respeito de Higiene, considera que os locais de comida de rua e venda ambulante são:*
S Sujos
Parcialmente sujos
○ Limpos

13. Quais os aspetos que gostaria de ver melhorados?

Higiene das instalações

Higiene dos manipuladores de alimentos

Qualidade sensorial dos alimentos

O Qualidade nutricional dos alimentos

O Outro 